Süleyman Demirel Üniversitesi Fen Edebiyat Fakültesi Fen Dergisi

Atıf için / For Citation: Z. TURHAN İRAK, M. POYRAZ, "[Ni(2-Benzimidazol-ilüre $\left.)_{2}(\text { etanol })_{2}\right]\left[\mathrm{NO}_{3}\right]_{2}$ Bileşiğinin Yoğunluk Fonksiyoneli Teorisi Kullanılarak Yapılan Bazı Kuantum Kimyasal Hesaplamaları", Süleyman Demirel Üniversitesi Fen Edebiyat Fakültesi Fen Dergisi, 14(2), 203-212, 2019.

\title{
[Ni(2-Benzimidazol-il-üre $\left.)_{2}(\text { etanol })_{2}\right]\left[\mathrm{NO}_{3}\right]_{2}$ Bileşiğinin Yoğunluk Fonksiyoneli Teorisi Kullanılarak Yapılan Bazı Kuantum Kimyasal Hesaplamaları
}

\author{
Zeynep TURHAN IRAK ${ }^{* 1}$, Mehmet POYRAZ ${ }^{2}$ \\ ${ }^{1} I \check{g}$ drr Üniversitesi, Mühendislik Fakültesi, Çevre Mühendisliği Bölümü, 76000, Iğdır, Türkiye \\ ${ }^{2}$ Afyon Kocatepe Üniversitesi, Fen Edebiyat Fakültesi, Kimya Bölümü, 03200, Afyonkarahisar, Türkiye \\ *yazışllan yazar e-posta: zeynepsilanturhan@ hotmail.com
}

(Alınış / Received: 12.04. 2019, Kabul / Accepted: 29.07. 2019, Yayımlanma / Published: 30.11.2019)

Özet: $\mathrm{Bu}$ çalışmada, moleküler yapısı tek bir kristal X-1şınım yöntemi ile belirlenen [Ni(2Benzimidazol-il-üre $\left.)_{2}(\text { etanol })_{2}\right]\left[\mathrm{NO}_{3}\right]_{2}$ bileșiğinin teorik hesaplamaları yapılmıştır. Teorik hesaplamalarda elde edilen optimize geometri, dipol moment gibi birçok özellik deneysel değerlere oldukça yakın sonuçlar göstermiştir. Bunların dışında bileşiğin gaz fazında sınır orbitalleri, hesaplanan sınır orbitallerin enerjilerinden elektronik yapı tanımlayıcıları, moleküler elektrostatik potansiyel haritası, doğrusal olmayan optik özellikleri hesaplanmış ve yorumlanmıștır. Çalıșmanın birçok organometal kompleksin moleküler özelliğine 1șık tutacağı düşünülmektedir. Yoğunluk Fonksiyonel Teorisi (YFT), Gaussian 09W programı kullanılarak hesaplama yöntemi olarak seçilmiştir ve kompleks için B3LYP/LANL2DZ seviyesinde optimize edilmiş gaz faz kuantum mekaniksel verileri elde edilmiştir.

Anahtar kelimeler: Benzimidazol-il-üre, Moleküler parametreler, YFT

\section{Some Quantum Chemical Calculations of the [Ni (2-Benzimidazoly-urea) ${ }_{2}$ $\left.(\text { ethanol })_{2}\right]\left[\mathrm{NO}_{3}\right]_{2}$ Compound Using Density Functional Theory}

\begin{abstract}
The theoretical calculations of the $\left[\mathrm{Ni}(2 \text {-Benzimidazoly-urea })_{2}(\text { ethanol })_{2}\right]\left[\mathrm{NO}_{3}\right]_{2}$ compound, whose molecular structure was determined with a single crystal X-ray diffraction method, was conducted. Many properties such as optimized geometry and dipole moment obtained in theoretical calculations showed very approximate to experimental values. Apart from these, the frontier orbitals of the compound in the gas phase, the electronic structure parameters from the energies of the calculated frontier orbitals, molecular electrostatic potential map, non-linear optical properties were calculated and interpreted. It is thought that the study will shed light on the molecular property of many organometal complexes. The Density Functional Theory (DFT) was selected as a computational method using the Gaussian 09W program, and the optimized gase phase quantum mechanical data for the complex was obtained in the B3LYP/LANL2DZ level with gen keyword.
\end{abstract}

Keywords: Benzimidazolyl-urea, Molecular parameters, DFT

\section{Giriş}

Benzimidazol ve türevleri ile ilgili çalışmalar son yıllarda oldukça ilgi kazanmıştır. Bu ilginin ana nedenlerinden biri benzimidazol ve türevi olan bileşiklerin özellikle kanser tedavisindeki etkilerinden kaynaklanmaktadır. Benzimidazol türevleri anti- viral [1], anti- kanser [2], anti-fungal [3], anti-mikrobiyal ve antibakteriyel [4-6] özelliklerinden 
dolayı yaygın olarak çalışılan ve kimyasal bakımdan dayanıklı, farklı fizyolojik özelliklere sahip önemli heterohalkalı bileşiklerdir.

Benzimidazoller ve benzimidazollerin çeşitli metal tuzları, uygun olan çözeltiler içerisinde, nötr ortamda benzimidazol-metal komplekslerini meydana getirirler [7-9]. Ayrıca, benzimidazol halkası içeren metal kompleksleri organik sentezlerde katalitik özellikler göstermektedir [10]. Benzimidazol türevleri korozyon yavaşlatıcısı olarak da kullanılmaktadır [11]. Tüm bunların yanında bazı benzimidazol türevlerinin lüminesans özellikleri de belirlenmiştir [12].

Üre türevleri yüksek antioksidan özelliklerinden dolayı adenokarsinom hücre serileri, MCF-7 hücrelerine, karşı güçlü antiproliferatif etki göstermektedirler. Üre türevlerinin lipid peroksidasyonunda inhibitör etki gösterdiği de bilinmektedir [13]. 2-benzimidazolil-ure türevleri ise tübülin inhibitörleri olarak bilinir [14]. Gümüş(I), bakır(II), çinko(II) ve nikel(II) metal iyonlarının 2-benzimidazol-il-ure kompleksleri sentezlenmiş ve antikanser etkileri araştırılmıştır [15-17].

Günümüzde kullanılan teorik hesaplama yöntemleri, sentezlenmiş/sentezlenmemiş veya sentezlenememiş, bunların dişında gerçek koşullarda oluşamayacak bileşikler için bile rahatça uygulanabilmektedir ve istenen sonuçlar alınabilmektedir. Hesaplama yöntemlerinden Yoğunluk Fonksiyoneli Teorisi (YFT) elektronik yap1 metotlarında kuantum mekaniksel yasaları kullanılır. Teorik modellemede YFT yaygın bir kullanıma sahiptir [18]. Literatürdeki var olan kaynaklar, YFT' nin bileşiklerin optimize edilmiş geometrisinin ve bu geometrinin dipol momentinin ve titreşim frekansları gibi birçok özelliğini de elde etmede deneysel değerlere oldukça yakın sonuçlar verdiğine işaret etmektedir [19].

$\mathrm{Bu}$ çalışmada, X-ışını kırınımı deneyi ile moleküler yapısı belirlenen [Ni(2benzimidazol-il-üre $\left.)_{2}(\text { etanol })_{2}\right]\left[\mathrm{NO}_{3}\right]_{2} \quad$ [17] bileşiğinin teorik hesaplamaları YFT yöntemiyle yapılmıştır ve hesaplanan teorik verilerle deneysel veriler karşılaştırılarak yorumlanmıştır. Ayrıca molekülün doğrusal olmayan optik özellikleri incelenmiştir.

\section{Materyal ve Metot}

Yapılan çalışmada, ab-initio, moleküler mekanik ve yarı-deneysel yöntemleri içeren, kapsamlı ve çok sayıda temel set seçeneğine sahip olan Gaussian 09W programı kullanılmıştır [20]. Gaussian 09W programıyla moleküllerin teorik kızılötesi (IR) ve UV-VIS spektrumları, NMR kimyasal kayma değerleri ve manyetik titreşim şiddetleri, kimyasal sertlik, kimyasal yumuşaklık, iyonlaşma enerjileri, elektron ilgisi, elektronegatiflik ve hiperkutuplanabilirlik gibi birçok özellik hesaplanabilir. Teorik olarak yapılan bütün hesaplama işlemlerinde moleküllerin veya atomların temel hali $\left(S_{0}\right)$ ya da uyarılmış hali $\left(S_{1}\right)$ kullanılabilir [20, 21]. Tüm yapıları geometrik optimizasyonlar1 MM2 metodu, yar1-ampirik PM3 metodu ve YFT metotuyla hesaplandıktan sonra frekansların negatif değer vermediği kontrol edilir ve böylece en uygun metot seçilebilmektedir [22].

Tüm teorik hesaplamalar Gaussian 09W programı kullanılarak YFT içerisinde Becke'nin üç parametreli enerji fonksiyoneli B3LYP hibrit yaklaşımı seçilerek yapılmıştır $[23,24]$. Kompleks gen keyword ile B3LYP/LANL2DZ düzeyinde optimize edilerek gaz fazındaki kuantum mekaniksel veriler elde edilmiştir [20,25]. Geometri optimizasyonu yapılan yapının öncelikle elektronik enerjisi, dipol momenti, en yüksek 
işgal edilmiş molekül orbitali (HOMO), en düşük işgal edilmemiş molekül orbitali (LUMO) ve $\Delta \mathrm{E}\left(\mathrm{E}_{\text {lumo }}-\mathrm{E}_{\text {homo }}\right)$ enerji değeri bulunmuştur. Moleküler elektrostatik potansiyel haritası (MEP) çıkartılmıştır. Doğrusal olmayan optik özellikler (NLO) için; tek nokta enerji hesabı kullanılarak polar hesapları yapılmış, kutuplanabilirlik $(\alpha)$, hiperkutuplanabilirlik $(\beta)$ değerleri hesaplanmıştır ve elde edilen veriler yorumlanmıştır.

\section{Bulgular}

\subsection{Kristal Yapıdan Elde Edilen Optimize Geometri}

Geometri optimizasyonu kararlı hal geometrisinin hesaplanmasıdır. Moleküllerdeki yapısal ve konumsal değişiklikler molekülün enerjisinde ve diğer özelliklerinde değişikliklere neden olur. Molekülün geometri optimizasyon hesabıyla yaklaşık en kararlı yapısı elde edilir. Böylece minimum enerjili sabit moleküler yapı bulunur [22].

[Ni(2-Benzimidazol-il-üre $\left.)_{2}(\text { etanol })_{2}\right]\left[\mathrm{NO}_{3}\right]_{2}$ bileşiğinin moleküler geometrisi doğrudan $\mathrm{X}$-1şını kırınım deneyi sonucundan alınmıştır. X-1şını kırınım deneyi sonuçlarından bileşiğin oktahedral yapıda kristallendiği görülmüştür (Şekil 1a) [17]. Bileşiğinin üç boyutlu yaklaşık geometrisi GaussView 5.0 programına [26] aktarılmış ve kompleks bileşiğin teorik hesaplamaları birim hücreden alınan tek bir molekül yapı üzerinden yürütülmüştür. Gaussian $09 \mathrm{~W}$ programı kullanılarak Yoğunluk Fonksiyonu Teorisi (YFT) metotunda B3LYP/LANL2DZ temel setiyle optimizasyon hesab1 yapılarak, bileşikteki her bir atomun minimum enerjili yapısı belirlenmiştir [27] (Şekil 1b).

YFT yöntemini kullanarak moleküler toplam enerji ve kompleksin sınır orbital (FMOs) enerjileri, B3LYP/LANL2DZ setinde 29 döngü hesaplamasından sonra kazanılmıştır. Kompleksin toplam enerjisi -2 247.7674 a.u'dur.

Kompleks bileşiğin X-ışını kırınım deneyi sonuçlarından elde edilen ve optimize edilerek teorik olarak hesaplanan geometriden yararlanarak seçilmiş bağ uzunlukları ve bağ açıları Tablo 1'de karşılaştırmalı olarak verilmiştir.

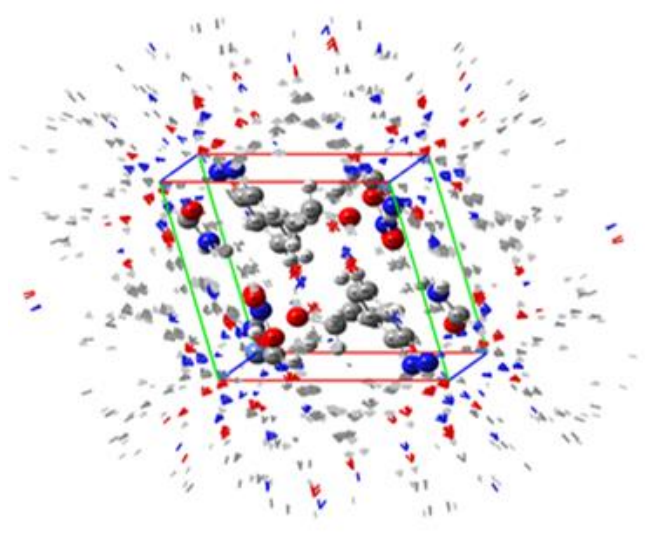

(a)

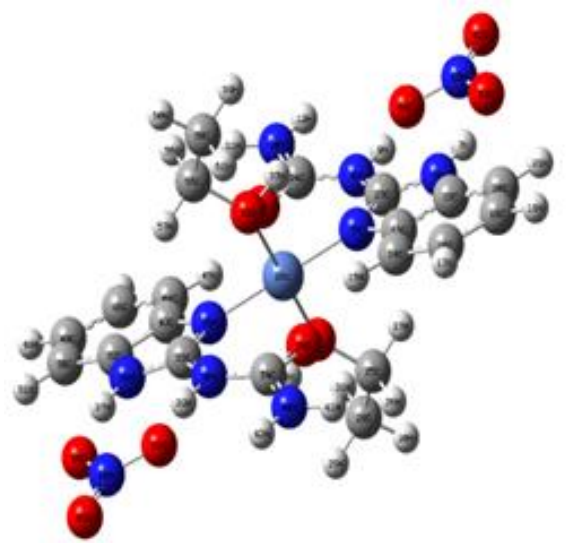

(b)

Şekil 1. (a) Molekülün X-1şını kırınım deneyi ile elde edilen geometrisi [17] (b) Molekülün B3LYP/LANL2DZ ile teorik olarak elde edilen geometrisi. 
Tablo 1. $\left[\mathrm{Ni}(2-B e n z i m i d a z o-i l-u r e)_{2}(\text { etanol })_{2}\right]\left[\mathrm{NO}_{3}\right]_{2}$ bileğinin $\mathrm{X}$-1şını kırınımı ve YFT yöntemleri kullanılarak elde edilmiş seçilmiş bağ uzunlukları $(\AA \hat{)})$ ve bağ açıları $\left(^{\circ}\right)$

\begin{tabular}{ccc}
\hline & $\begin{array}{c}\text { X-Işını } \\
\text { Kırınımı }^{(17)}\end{array}$ & YFT \\
\hline Bă̆ Uzunluğu & & \\
\hline $1 \mathrm{Ni}-4 \mathrm{O}(34 \mathrm{O})$ & $2.0419(14)$ & 1.90696 \\
$1 \mathrm{Ni}-2 \mathrm{O}(32 \mathrm{O})$ & $2.0930(15)$ & 2.83352 \\
$1 \mathrm{Ni}-5 \mathrm{~N}(35 \mathrm{~N})$ & $2.0833(16)$ & 1.92290 \\
2O(32O)-25C(55C) & $1.447(3)$ & 1.47455 \\
$4 \mathrm{O}(34 \mathrm{O})-24 \mathrm{C}(54 \mathrm{C})$ & $1.232(2)$ & 1.29148 \\
$5 \mathrm{~N}(35 \mathrm{~N})-13 \mathrm{C}(43 \mathrm{C})$ & $1.411(2)$ & 1.42236 \\
Bă̆ Açısı & & \\
$4 \mathrm{O}-1 \mathrm{Ni}-34 \mathrm{~N}$ & 180.0 & 179.98162 \\
$5 \mathrm{~N}-1 \mathrm{Ni}-35 \mathrm{~N}$ & 180.0 & 179.99658 \\
$2 \mathrm{O}-1 \mathrm{Ni}-32 \mathrm{O}$ & 180.0 & 179.96998 \\
$4 \mathrm{O}-1 \mathrm{Ni}-5 \mathrm{~N}$ & $87.19(6)$ & 89.75260 \\
$4 \mathrm{O}-1 \mathrm{Ni}-35 \mathrm{~N}$ & $92.81(6)$ & 90.24414 \\
$34 \mathrm{O}-1 \mathrm{Ni}-5 \mathrm{~N}$ & $92.81(6)$ & 90.24848 \\
$34 \mathrm{O}-1 \mathrm{Ni}-35 \mathrm{~N}$ & $87.19(6)$ & 89.75477 \\
\hline
\end{tabular}

Teorik olarak bulunan optimize geometri X-1şını kırınımı deneyinden elde edilen geometriyle uyum göstermiştir. Tablo 1'e bakıldığında deneysel ve teorik bağ uzunlukları ve bağ açıları arasında bazı ufak farklılıklar göze çarpmaktadır. Bu farklılıkların sebebi; teorik hesaplamaların hem gaz fazında yapılmış olması hem de birim hücreden alınan izole tek molekül üzerinden yapılması ve dolayısıyla olası diğer etkileşimlerin ihmal edilmesi olarak açıklanabilir.

\subsection{Moleküler Elektrostatik Potansiyel (MEP)}

Moleküler elektrostatik potansiyel; moleküler sistemin yük dağılımı ile birim pozitif yük arasında olan etkileşim enerjisi olarak tanımlanabilir. MEP haritası üzerinde en negatif potansiyel kırmızı renkle gösterilirken en pozitif potansiyel mavi renkle gösterilir [28]. Molekül etkileşimde moleküllerin MEP'i anahtar rol oynar. Bir molekülün MEP'inin en negatif olduğu bölge elektrofilik atağa en yatkın bölgeyken; en pozitif olduğu bölge nükleofilik atağa en yatkın bölge olarak tanımlanabilir [29].

Hesaplama sonucunda elde edilen $\quad\left[\mathrm{Ni}(2 \text {-Benzimidazol-il-ure })_{2}(\mathrm{etanol})_{2}\right]\left[\mathrm{NO}_{3}\right]_{2}$ molekülüne ait MEP haritası dikkate alındığında (Şekil 2), en negatif bölge -0.089 a.u değeriyle nitrat grubu oksijenleri üzerine yerelleşmiş olan bu bölgenin elektrofilik atağa en yatkın bölge olduğu söylenebilir. Diğer taraftan en pozitif bölgenin ise 0.090 a.u. değeriyle ürenin hidrojenleri etrafina yerelleştiği görülmektedir; bu durum bölgenin herhangi bir nükleofilik atak için en yatkın bölge olduğu söylenebilir. 


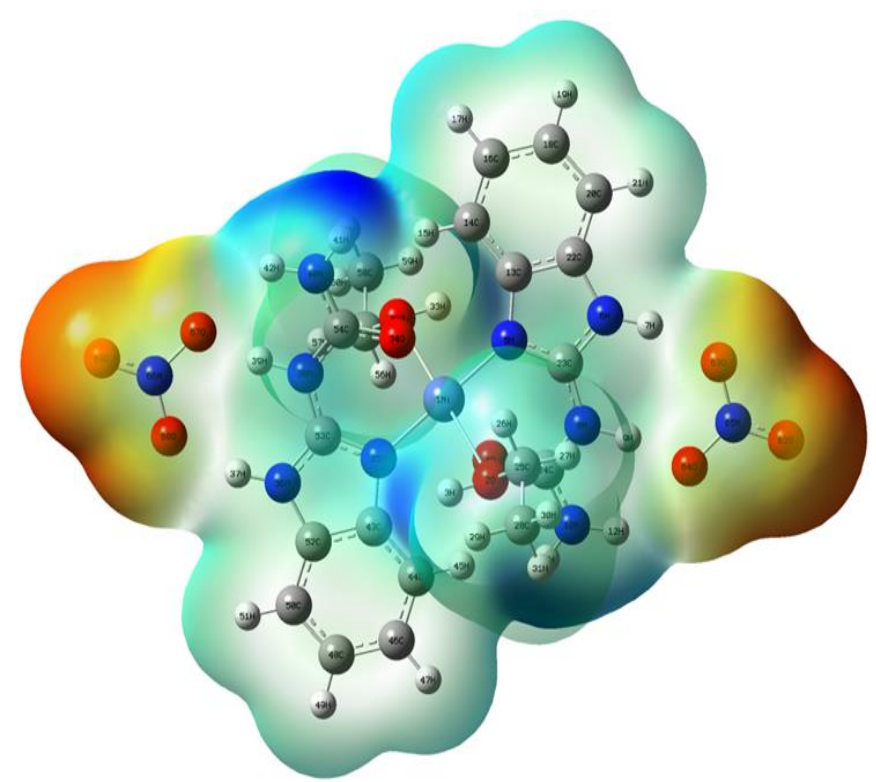

Şekil 2. $\left[\mathrm{Ni}(2-\mathrm{Benzimidazol-il-ure})_{2}(\text { etanol })_{2}\right]\left[\mathrm{NO}_{3}\right]_{2}$ 'in YFT ile hesaplanan MEP haritas1.

\subsection{Sinır Orbitaller (FMOs) ve Elektronik Özellikler}

HOMO ve LUMO enerjileri, en çok bilinen kuantum kimyasal parametrelerdir. Sınır orbitaller olarak da bilinen bu orbitaller başta kimyasal reaksiyonlar olmak üzere bir molekülün hem elektronik hem de optik özelliklerinin belirlenmesinde önemli rol oynamaktadır [30]. Teorik hesaplama sonucunda 158 tane işgal edilmiş orbitale sahip olduğu belirlenmiştir. HOMO ve LUMO yüzeyleri ve enerjileri Şekil 3 'de verilmiştir.

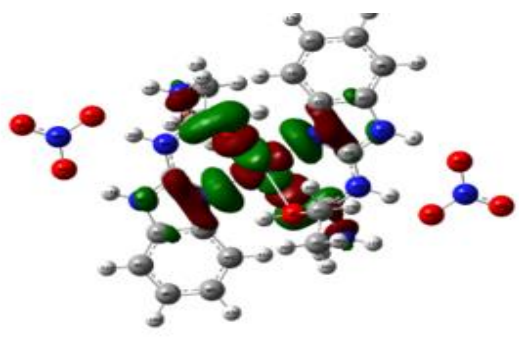

$E_{\text {LUMO }}=-2.885 \mathrm{eV}$
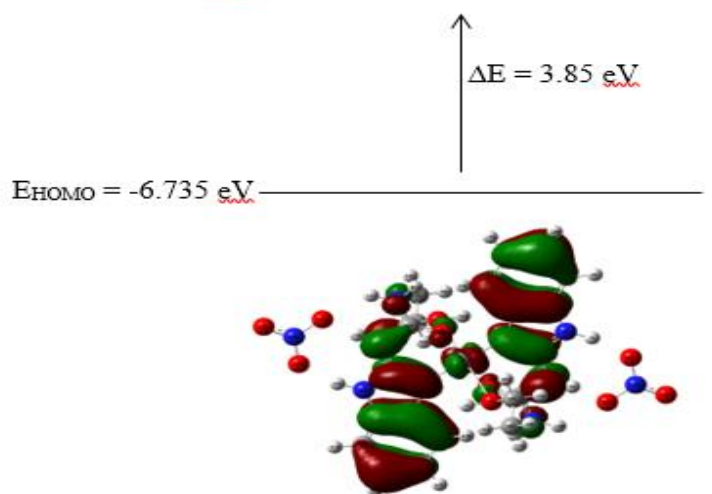

Şekil 3. Molekülün B3LYP/LANL2DZ'ye göre hesaplanan HOMO-LUMO yüzeyleri ve enerjileri

Sınır molekül orbital kuramına göre, bir geçis halinin oluşması reaksiyona giren sınır orbitaller (HOMO ve LUMO) arasındaki bir etkileşim sebebiyledir [31]. HOMO enerjisi doğrudan iyonlaşma potansiyeliyle ve LUMO enerjisi ise doğrudan elektron ilgisiyle ilişkilidir. HOMO-LUMO bant aralığı, yani HOMO ve LUMO enerjileri arasındaki fark, önemli bir kararlılık indeksidir [32]. Büyük bir HOMO-LUMO 
boşluğu, kimyasal reaksiyonlarda düşük reaktiflik anlamında olup, molekülün yüksek kararlılığını gösterir [33,34]. Çalışmada bileşiğin $\Delta \mathrm{E}$ değeri $3.85 \mathrm{eV}$ olarak hesaplanmıştır. Hesaplanan bu enerji aralığı değeri bize molekülün yüksek kinetik kararlılığa ve düşük kimyasal reaktiviteye sahip olduğunu gösterir.

Koopman teorisine [35] göre iyonlaşma potansiyeli (I), I= - $E_{\text {homo }}$ ve elektron ilgisi (A), $\mathrm{A}=-\mathrm{E}_{\text {lumo }}$ 'dur. Nötral moleküllerin HOMO-LUMO enerjilerinden molekülün elektronegatiflik $(\chi)$, sertlik ( $\eta$ ve yumuşaklık $(\sigma)$ değerleri (Eşitlik 1-3) hesaplanmıştır [36] ve sonuçlar Tablo 2'de verilmiştir.

$$
\begin{gathered}
\chi=(I+A) / 2 \\
\eta=(I-A) / 2 \\
\sigma=1 / \eta
\end{gathered}
$$

Tablo 2. Molekülün hesaplanan dipol moment $(\mu)$, iyonlaşma potansiyeli (I), elektron ilgisi (A), elektronegatiflik $(\chi)$, sertlik $(\eta)$ ve yumuşaklık $(\sigma)$ değerleri

\section{B3LYP/LANL2DZ}

\begin{tabular}{cc}
\hline$\mu$ (debye) & 0.1111 \\
$\mathrm{I}(\mathrm{eV})$ & 6.735 \\
$\mathrm{~A}(\mathrm{eV})$ & 2.885 \\
$\chi(\mathrm{eV})$ & 4.81 \\
$\eta(\mathrm{eV})$ & 1.925 \\
$\sigma\left(\mathrm{eV}^{-1}\right)$ & 0.52 \\
\hline
\end{tabular}

\subsection{Doğrusal Olmayan Optik Özellikler (NLO)}

Doğrusal olmayan optik (NLO) özellik gösteren moleküller ve bu moleküllerin uygulamaları güncel bir araştırma konusudur ve NLO malzemeleri yarı iletken tabakalı yapılar olarak kategorize edilir. Bu nedenle, inorganik, organik ve organometalik moleküler sistemlerin birçok çeşidi NLO aktivitesi için incelenmektedir [37, 38]. Optik özellikler, maddenin içerisindeki elektronların elektriksel alana gösterdikleri tepki olarak ifade edilmektedir. Organik tabanlı maddelerde ise optik özellikler kutuplanabilirlik ile tanımlanmaktadır. Hiperkutuplanabilirlik ise maddenin doğrusal olmayan optik özelliklerinin performansını etkileyen bir niceliktir [39].

Molekülün dipol momentinin yeniden şekillenmesi, uygulanan bir dış elektrik alana karşı verdiği tepkiyle olur.

$$
\begin{gathered}
\mu_{(t)}=\mu_{0}+\alpha E(t)+1 / 2 \beta E 2(t)+1 / 6 \gamma E 3(t)+\ldots \ldots . . \\
\alpha=1 / 3\left(\alpha_{x x}+\alpha_{y y}+\alpha_{z z}\right) \\
\beta=\left[\left(\beta_{x x x}+\beta_{x y y}+\beta_{x z z}\right) 2+\left(\beta_{y y y}+\beta_{x x y}+\beta_{y z z}\right) 2+\left(\beta_{z z z}+\beta_{x x z}+\beta_{y y z}\right) 2\right]^{1 / 2}
\end{gathered}
$$

Eşitlikte $\mu 0$, molekülün kalıcı dipol momenti, $\sigma$ moleküler kutuplanabilirlik, $\beta$ birinci derecen hiperkutuplanabilirlik ve $\gamma$ ise ikinci dereceden hiperkutuplanabilirlik olarak tanımlanmaktadır. Uygulanan elektrik alan zayıf olduğunda moleküler kutuplanabilirlik önemliyken, elektrik alan şiddetli olduğunda doğrusal olmayan özellik olan 
hiperkutuplanabilirlik önemli bir hal alır [40]. Kutuplanabilirlik ile HOMO-LUMO enerji farkı aralığında karşılıklı bir ilişki söz konusudur [41]. $\Delta \mathrm{E}$ aralığı ne kadar düşük olursa elektron dağılımı o kadar kolay yönlenir ve kutuplanma büyük olur. Kutuplanabilirlik ile arasında ilişki olan $\Delta \mathrm{E}$ hiperkutuplanabilirlik için yeterli düzeyde belirleyici değildir.

NLO özelliklerini hesapsal olarak öngörmek için dipol moment $(\mu)$, kutuplanabilirlik $(\alpha)$ ve hiperkutuplanabilirlik $(\beta)$ değerlerinden faydalanılır [42]. Doğrusal olmayan optik özellikler hesaplanırken; ortalama lineer kutuplanabilirlik için, ve toplam statik hiperkutuplanabilirlik için, yukarıdaki eşitlikler kullanılmıştır.

Tablo 3. Gaz fazında molekülün B3LYP/LANL2DZ kullanılarak hesaplanan kutuplanabilirlik $(\alpha)$ ve hiperkutuplanabilirlik $(\beta)$ değerleri verilmiştir

\begin{tabular}{cc}
\hline & B3LYP/LANL2DZ \\
\hline $\boldsymbol{\alpha}_{\mathbf{x x}}$ & 430.611267 a.u \\
$\boldsymbol{\alpha}_{\mathbf{y y}}$ & 448.054238 a.u \\
$\boldsymbol{\alpha}_{\mathbf{z z}}$ & 199.682277 a.u \\
$\boldsymbol{\alpha}$ & $53.27 \times 10-{ }^{24} \mathrm{esu}$ \\
$\boldsymbol{\beta}_{\mathbf{x x x}}$ & 0.251561 a.u \\
$\boldsymbol{\beta}_{\mathbf{x y y}}$ & $-0.138935 \mathrm{a} . \mathrm{u}$ \\
$\boldsymbol{\beta}_{\mathbf{x z z}}$ & 0.062637 a.u \\
$\boldsymbol{\beta}_{\mathbf{y y y}}$ & 0.243249 a.u \\
$\boldsymbol{\beta}_{\mathbf{x x y}}$ & 0.107970 a.u \\
$\boldsymbol{\beta}_{\mathbf{y z z}}$ & 0.455809 a.u \\
$\boldsymbol{\beta}_{\mathbf{z z z}}$ & $-0,130406$ a.u \\
$\boldsymbol{\beta}_{\mathbf{x x z}}$ & $-0,013945$ a.u \\
$\boldsymbol{\beta}_{\mathbf{y y z}}$ & $-0,018789$ a.u \\
$\boldsymbol{\beta}$ & $0.0073 \times 10^{-30} \mathrm{esu}$ \\
\hline
\end{tabular}

Tablo 3'de gaz fazında molekülün B3LYP/LANL2DZ kullanılarak hesaplanan kutuplanabilirlik $(\alpha)$ ve hiperkutuplanabilirlik $(\beta)$ değerleri verilmiştir. Kutuplanabilirlik ve hiperkutuplanabilirlik değerlerleri atomik biriminden (a.u) elektrostatik birime (esu) $\left(\alpha: 1\right.$ a.u. $=0.1482 \times 10^{-24}$ esu, $\beta: 1$ a.u. $\left.=8.6393 \times 10^{-33} \mathrm{esu}\right)$ dönüştürülmüştür [41].

Tablodan görüldüğ̈̈ gibi gaz fazında $(\varepsilon=1)$ hesaplanan [Ni(2-Benzimidazolure $\left.)_{2}(\text { etanol })_{2}\right]\left[\mathrm{NO}_{3}\right]_{2}$ molekülünün kutuplanabilirlik değeri $(\alpha) 53.27 \times 10^{-24}$ esu ve hiperkutuplanabilirlik değeri $(\beta) 0.0073 \times 10^{-30}$ esu olarak bulunmuştur.

\section{Sonuç ve Yorum}

Bu çalışmada, X-ışını kırınımı yöntemiyle daha önce deneysel olarak çalışılmış [17] [Ni(2-Benzimidazol-il-ure $\left.)_{2}(\mathrm{etanol})_{2}\right]\left[\mathrm{NO}_{3}\right]_{2}$ molekülünün yapısal, moleküler, elektronik ve optik özellikleri YFT yöntemiyle teorik olarak incelenmiştir. Molekülün optimize geometrisine, bağ uzunluklarına ve açılarına bakıldığında YFT' nin deneysel olarak bulunan sonuçlarla bazı ufak farklılıklar dışında uyumlu olduğu gözlenmiştir. Bu ufak farklılıklar incelenen molekülün gaz fazında izole edilmiş tek bir molekül olarak alınmasından ve diğer moleküler etkileşmelerin dikkate alınmamasından kaynaklanmaktadır. Molekülün MEP haritasına bakıldığında; ürenin benzimidazol halkasına bağlı olmayan azotunun hidrojenlerinin nükleofilik atak için, nitrat grubu oksijenlerinin ise herhangi bir elektrofilik atak için en uygun bölgeler olduğu söylenebilir. $3.85 \mathrm{eV}$ olarak hesaplanan HOMO-LUMO enerji aralığ1, molekülün yüksek kinetik kararlılığa ve düşük kimyasal reaktiviteye sahip olduğunu göstermektedir. 
Çalışmada hesaplanan HOMO-LUMO enerji farkları ve bu enerji farklarından moleküler parametreler (I; İyonlaşma potansiyeli, A; elektron ilgisi, $\eta$; moleküler sertlik, $\sigma$; moleküler yumuşaklık ve $\chi$; elektronegatiflik) değerleri belirlenmiştir Yüksek sertlik düşük yumuşaklık molekülün kararlığının göstergesidir.

Çalışmada son olarak incelenen molekülün doğrusal olmayan optik özellikleri belirlendi. Hiperkutuplanabilirlik değerinin teorik olarak elde edilmesi, moleküler yap1 ile doğrusal olmayan optik özellikler arasındaki ilişkinin anlaşılmasında oldukça kullanışlı ve yaygın bir yöntem olduğu bilindiğinden, bir moleküler sistemin $\beta$ parametresinin yüksek değerde olması, konjuge $\pi$ sistemi boyunca elektronların hareketinden kaynaklanan molekül içi yük transferi ile bağdaştırılır. Doğrusal olmayan optik özellik gösteren bileşiklerde ürenin hiperkutuplanabilirlik değeri referans olarak kullanılmaktadır. $\quad\left[\mathrm{Ni}(2 \text {-Benzimidazol-il-ure })_{2}(\mathrm{etanol})_{2}\right]\left[\mathrm{NO}_{3}\right]_{2}$ molekülünün teorik olarak bulunan hiperkutuplanabilirlik değerinin, ürenin $0.77 \times 10^{-30}$ esu olarak bilinen hiperkutuplanabilirlik değerinden daha düşük olduğu görülmüştür. Bulunan değer çalışılan molekülün, molekül içi yük transferinin üreye göre az olduğunu düşündürür ki; bu da bileşiğin doğrusal olmayan optik malzemeler için aday olamayacağını göstermektedir.

\section{Kaynakça}

[1] M.M. Ramla, and M.A. Omar, "Discovery and optimization of pyrrolo[1,2-a] pyrazinones leads to novel and selective inhibitors of PIM kinases," Bioorg. Med. Chem., 14 (21), 7324-7332, 2006.

[2] D. Pathak, N. Siddiqui, B. Bhrigu, W. Ahsan, and M. S. Alam, "Benzimidazoles: A new profile of biological activities," Der Pharmacia Lettre, 2(2), 27-34, 2010.

[3] H. Goker, C. Kus and D.W. Boykin, "Synthesis of some new 2-substituted-phenyl-1Hbenzimidazole-5-carbonitriles and their potent activity against candida species," Bioorg. Med. Chem., 10, 2589-96, 2002.

[4] H. Goker, C. Kus and D.W. Boykin, "Synthesis and potent antibacterial activity against MRSA of some novel 1,2- disubstituted-1H-benzimidazole-N-alkylated-5- carboxamidines," Eur. J. Med. Chem., 40, 1062, 2005.

[5] M. Andrzejewska and M.L. Yepez, "Synthesis, antiprotozoal and anticancer activity of substituted 2trifluoromethyl- and 2-pentafluoroethylbenzimidazoles," Eur. J. Med. Chem., 37, 973, 2002.

[6] S. Ozden, D. Atabey, and H. Goker, "Synthesis and potent antimicrobial activity of some novel methyl or ethyl 1H-benzimidazole-5-carboxylates derivatives carrying amide or amidine groups," Bioorg. Med. Chem., 13, 1587-97, 2005.

[7] R. Curini, S. Materazzi, G. D' Ascenzo, and G. De Angelis, "Thermal behavior of biological interesting coordination compounds of benzimidazole with divalent metal ions," Thermochim. Acta, 161(2), 201-374, 1990.

[8] G. Xue, J. Zhang, G. Shi, Y. Wu, and B. Shuen, "Spectroscopic studies on the polymerization of benzimidazole with metallic copper," J. Chem. Soc., 2, 33-36, 1989.

[9] N.T. Abdel Ghani, and A.M. Mansour, "Palladium (II) and platinum (II) complexes containing benzimidazole ligands: Molecular structures, vibrational frequencies and cytotoxicity," $J$ Mol. Struct., 991,108-126, 2011.

[10] H. Küçükbay, B. Çetinkaya, S. Guesmi, and P.H. Dixneuf, "New (Carbene) ruthenium-arene complexes: Preparation and uses in catalytic synthesis of furans,"Organometallics, 15, 2434-2439, 1996.

[11] A. Popova, M. Christov, and A. Zwetanova, "Effect of the molecular structure on the inhibitor properties of azoles on mild steel corrosion in 1 M hydrochloric acid," Corros. Sci., 49(5), 2131 2143, 2005.

[12] L. Cireş, H. Ofenberg, T. Nicolaescu, C. Crăiţăa, and A. Pollet, "Substituent influence on the fluorescence spectra of 2, 3-diphenylbenzo [b] furan derivatives," J. Luminescence, 79(2), 91-96, 1998. 
[13] I. Perkovic, M. Antunovic, I. Marijanovic, K. Pavic, K. Ester, M. Kralj, J. Vlainic, I. Kosalec, D. Schols, D. Hadjipavlou-Litina, E. Pontiki, and B. Zorc, "Novel urea and bis-urea primaquine derivatives with hydroxyphenyl or halogenphenyl substituents: Synthesis and biological evaluation," Eur. J. Med. Chem., 124, 622-636, 2016.

[14] W. Wang, D. Kong, H. Cheng, L. Tan, Z. Zhang, X. Zhuang, H. Long, Y. Zhou, Y. Xu, X. Yang, and K. Ding, "New benzimidazole-2-urea derivates as tubulin inhibitors," Bioorg. Med. Chem. Lett., 24, 4250-4253, 2014.

[15] M. Poyraz, H. Berber, C.N. Banti, N. Kourkoumelis, M.J. Manos, and S.K. Hadjikakou, "Synthesis characterization and biological activity of mixed ligand silver(I) complex of 2-benzimidazolylurea and triphenylphosphine," Polyhedron, 128, 95-103, 2017.

[16] M. Poyraz, M. Sari, and C.N. Banti, S.K. Hadjikakou, "Synthesis, characterization and biological activities of copper(II) complex of 2-Benzimidazolyl-urea and the nitrate salt of 2-Benzimidazolylurea," J. Mol. Struct., 1146, 809-813, 2017.

[17] C.N. Banti, M. Poyraz, I. Sainis, M. Sari, G.Rossos, N. Kourkoumelis, and S.K. Hadjikakou, "The periodic table of urea derivative: small molecules of zinc(II) and nickel(II) of diverse antimicrobial and antiproliferative applications" Mol. Divers., 1 - 13, 2019.

[18] D. C. Young, Computational Chemistry. New York: Wiley-Interscience, 2011.

[19] F. De Proft, and P. Geerlings, "Conceptual and computational DFT in the study of aromaticity," Chem. Rev., 101(5), 1451- 1464, 2001.

[20] M.J. Frisch, G.W. Trucks, H.B. Schlegel, G.E. Scuseria, M.A. Robb, J.R. Cheeseman, G. Scalmani, V. Barone, B. Mennucci, G.A. Petersson, H. Nakatsuji, M. Caricato, Li X., H.P. Hratchian, A.F. Izmaylov, J. Bloino, G. Zheng, J.L. Sonnenberg, M. Hada, M. Ehara, K. Toyota, R. Fukuda, J. Hasegawa, M. Ishida, T. Nakajima, Y. Honda, O. Kitao, H. Nakai, T. Vreven, J. A. Montgomery, T.Jr. Vreven, J.E. Peralta, F. Ogliaro, M. Bearpark, J.J. Heyd, E. Brothers, N. Kudin, V.N. Staroverov, R. Kobayashi, J. Normand, K. Raghavachari, A. Rendell, J.C. Burant, S.S. Iyengar, J. Tomasi, M. Cossi, N. Rega, J.M. Millam, M. Klene, J.E. Knox, J.B. Cross, V. Bakken, C. Adamo, J. Jaramillo, R. Gomperts, R.E. Stratmann, O. Yazyev, A.J. Austin, R. Cammi, C.J. Pomelli, W. Ochterski, L.R. Martin, K. Morokuma, V.G. Zakrzewski, G.A. Voth, P. Salvador, J.J. Dannenberg, S. Dapprich, A.D. Daniels, O. Farkas, J.B. Foresman, J.V. Ortiz, J. Cioslowski, and D.J. Fox, Gaussian09, Wallingford, CT, USA: Gaussian. Inc.

[21] P.H. Gümüş, Ö. Tamer, D. Avc1, and Y. Atalay, “4-(Metoksimetil)-1,6-dimetil-2-okso-1,2dihidropiridin-3-karbonitril molekülünün teorik olarak incelenmesi," Sakarya Üniversitesi Fen Bilimleri Dergisi, 3, 303-311, 2015.

[22] Z. Turhan Irak, S. Gümüş, "Heterotricyclic compounds via click reaction: A computational study," Noble International Journal of Scientific Research, 1(7), 80-89, 2017.

[23] A.D. Becke, "Density-functional exchange energy approximation with correct asymptotic behavior," Physical Review A, 3098-3100, 1988.

[24] C. Lee, W.R. Yang, and Parr G, "Development of the Colle-Salvetti correlation-energy formula into a functional of the electron density," Phys. Rev. B, 785-789, 1998.

[25] J.B. Foresman, and Æ. Frisch, Exploring Chemistry with electronic structure methods (2nd ed.), Pittsburgh, PA: Gaussian Inc., 1996.

[26] R. Dennington, T. Keith, and J. Millam, GaussView Version 5, Shawnee Mission KS: Semichem Inc., 2009.

[27] Z. Turhan Irak, A. Altıkat, Z. Bingül, E. Öztürk, and A. Yiğit, "Determination of DFT method activities of some triazine derivatives as corrosion inhibitors," in Proc. $4^{\text {th }}$ Imcofe, Roma, 2017, pp. 309-310.

[28] C.J. Cramer, Essentials of computational chemistry. London: John Wiley and Sons, 2004, pp.596.

[29] I. N. Levine, Many- Electron Atoms: Quantum chemistry, New Jersey: Prentice- Hall Inc, 2000, pp.739.

[30] I. Fleming, Frontier Orbitals and Organic Chemical Reactions. London: J. Wiley and Sons, 1976, pp. 249.

[31] K. Fukui, Theory of Orientation and Stereoselection, New York: Springer-Verlag, 1975, pp. 420. 
[32] D.F.V. Lewis, C. Ioannides, and D.V. Parke, "Interaction of a series of nitriles with the alcoholinducible isoform of P450: Computer analysis of structure activity relationships," Xenobiotica, 24, 401-408,1994.

[33] R.G. Pearson, "Absolute electronegativity and hardness: Applications to organic chemistry," J. Org. Chem., 54, 1423-1430, 1989.

[34] Z. Zhou, and R.G. Parr, "Activation hardness: New index for describing the orientation of electrophilic aromatic substitution,” J. Am. Chem. Soc., 112, 5720-5724, 1990.

[35] L. Ji, Q.X. Zeng, M.L. Wei., L.W. Jin, Q.Y Zhong, "Koopmans Theorem for Large Molecular Systems within Density Functional Theory,"J. Phys. Chem., 110(43), 12005-12009, 2006.

[36] D.O. Isin, and N. Karakus, "Quantum chemical study on the inhibition efficiencies of some symtriazines as inhibitors for mild steel in acidic medium," J. Taiwan Inst. Chem. E., 50, 306-313, 2015.

[37] M. D. Aggarwal, J. Stephens, A. K. Batra, and R.B. Lal, "Bulk growth and characterization of semiorganic nonlinear optical materials,” J. Optoelectron. Adv. M., 5(3), 555- 562, 2003.

[38] J. Zyss, Molecular Non-linear Optics: Materials, physics and devices, Boston: Academic Press, 1994.

[39] J. Leszczynski, Non-Linear Optical Properties of Matter, Dordrecht, The Netherlands: Springer, 2006, pp. 676.

[40] Z. İrak, S. Gümüş, "Donör-akseptör gruplar içeren bazı heterosiklik bileşiklerin çizgisel olmayan özelliklerinin teorik olarak incelenmesi," Kafkas Üniversitesi Fen Bilimleri Enstitüsü Dergisi, 10(1), $1-8,2017$.

[41] A. Hinchliffe, M. H. J. Soscun, "Ab initio studies of the dipole polarizabilities of conjugated molecules, Part 2: Monocyclic azines,” Journal of Molecular Structure, 110 (2), 109- 120,1997.

[42] M. Govindarajan, S. Periandy, and K. Carthigayen, "FT-IR and FT-Raman spectra, thermo dynamical behavior, HOMO and LUMO, UV, NLO properties, computed frequency estimation analysis and electronic structure calculations on $\alpha$-bromotoluene," Spectrochim. Acta A., 97, 411422, 2012. 